\title{
Dysregulated IncRNA-miRNA-mRNA Network Reveals Patient Survival- Associated Modules and RNA Binding Proteins in Invasive Breast Carcinoma
}

OPEN ACCESS

Edited by:

Juan Xu,

Harbin Medical University, China

Reviewed by:

Meng Zhou,

Wenzhou Medical University, China

Kuixi Zhu,

University of Arizona, United States

*Correspondence:

Chunjie Jiang

chunjie.jiang@pennmedicine.

upenn.edu

Specialty section:

This article was submitted to

RNA,

a section of the journal

Frontiers in Genetics

Received: 03 September 2019 Accepted: 21 November 2019

Published: 15 January 2020

Citation:

Dong Y, Xiao Y, Shi $Q$ and Jiang C (2020) Dysregulated IncRNA-miRNAmRNA Network Reveals Patient

Survival-Associated Modules and RNA Binding Proteins in Invasive Breast Carcinoma.

Front. Genet. 10:1284.

doi: 10.3389/fgene.2019.01284
Yu Dong ${ }^{1}$, Yang Xiao ${ }^{2,3}$, Qihui Shi ${ }^{1}$ and Chunjie Jiang ${ }^{2,3 *}$

${ }^{1}$ Key Laboratory of Systems Biomedicine (Ministry of Education), Shanghai Center for Systems Biomedicine, Shanghai Jiao Tong University, Shanghai, China, ${ }^{2}$ Institute for Diabetes, Obesity, and Metabolism, Perelman School of Medicine at the University of Pennsylvania, Philadelphia, PA, United States, ${ }^{3}$ Division of Endocrinology, Diabetes, and Metabolism, Department of Medicine, Perelman School of Medicine at the University of Pennsylvania, Philadelphia, PA, United States

Breast cancer is the most common cancer in women, but few biomarkers are effective in clinic. Previous studies have shown the important roles of non-coding RNAs in diagnosis, prognosis, and therapy selection for breast cancer and have suggested the significance of integrating molecules at different levels to interpret the mechanism of breast cancer. Here, we collected transcriptome data including long non-coding RNA (IncRNA), microRNA (miRNA), and mRNA for 1,200 samples, including 1079 invasive breast carcinoma samples and 104 normal samples, from The Cancer Genome Atlas (TCGA) project. We identified differentially expressed IncRNAs, miRNAs, and mRNAs that distinguished invasive carcinoma samples from normal samples. We further constructed an integrated dysregulated network consisting of differentially expressed IncRNAs, miRNAs, and mRNAs and found housekeeping and cancer-related functions. Moreover, 58 RNA binding proteins (RBPs) involved in biological processes that are essential to maintain cell survival were found in the dysregulated network, and 10 were correlated with overall survival. In addition, we identified two modules that stratify patients into high- and low-risk subgroups. The expression patterns of these two modules were significantly different in invasive carcinoma versus normal samples, and some molecules were high-confidence biomarkers of breast cancer. Together, these data demonstrated an important clinical application for improving outcome prediction for invasive breast cancers.

Keywords: IncRNAs, RNA binding protein, miRNAs, integrative analysis, invasive breast carcinoma, biomarker 


\section{INTRODUCTION}

In women, breast cancer is the most commonly diagnosed cancer and accounts for $\sim 30 \%$ of new cancer diagnoses (Siegel et al., 2017). Great improvements have been achieved in diagnosis, surgery, and medical treatment for breast cancer in the past decades. From 1989 to 2016, the death rate for breast cancer dropped by $40 \%$ for female breast cancers in the United States. However, it has still been the second leading cause of cancer death in women in the last ten years (Siegel et al., 2017). Invasive breast carcinoma accounts for about $80 \%$ of breast cancer (Weigelt et al., 2008) and exhibits high heterogeneity in terms of morphology, clinical features, and prognosis (Milanovic et al., 2013), and the regulatory mechanisms at the genomic level still thus need to be unearthed.

Many studies have investigated the pathogenesis underlying breast cancer and have discovered diagnostic and prognostic markers. In 2006, a study reported altered expression patterns of microRNAs (miRNAs) during initiation and progression and their relationship with cancer diagnosis, staging, and prognosis (Calin and Croce, 2006). Another study investigated the expression of deregulated miRNAs in breast cancer and found correlations of altered miRNA expression with estrogen receptor expression, vascular invasion, and other clinicopathological characteristics (Iorio et al., 2005). Long non-coding RNAs (lncRNAs) represent a new class of non-coding RNAs that are at least 200 nucleotides in length and do not possess a clearly defined open reading frame (Ponting et al., 2009). lncRNAs are critical regulatory factors in cancer initiation and progression ( $\mathrm{Li}$ and Chen, 2013; Yang et al., 2014). The lncRNA DSCAM-AS1 holds a central position in estrogen receptor (ER)-regulated breast cancer and modulates tamoxifen resistance and tumor progression (Niknafs et al., 2016). Another lncRNA, MAGI2AS3, can target the Fas/FasL signaling pathway to suppress cell growth in breast cancer (Yang et al., 2018b). Furthermore, a 12lncRNA signature has been proposed that can be used to identify breast cancer patients at high risk of tumor recurrence, which could be utilized in clinic (Zhou et al., 2016b). Recently, some studies have shown that post-transcriptional regulatory networks can be regulated by molecules at multiple levels (Wei et al., 2017; Liu et al., 2019). By constructing a ceRNA network, a 10-lncRNA signature has been proposed that classified patients into highand low-risk subgroups with significantly different survival outcomes, highlighting the value of integrating data sets from multiple levels (Zhou et al., 2016a). Mir-21 and lncRNA AWPPH regulate cancer cell chemosensitivity and proliferation in triplenegative breast cancer (Liu et al., 2019). Mir-223 promotes breast cancer cell proliferation by targeting FOXO1 and provides a new potential tumor marker (Wei et al., 2017). The above results imply the significance of integrating molecules at different regulatory levels for interpreting the mechanism of breast cancer, especially in invasive breast carcinoma.

RNA-binding proteins (RBPs) are a type of proteins that bind RNA through its globular RNA-binding domains (RBDs) (Hentze et al., 2018). RBPs can bind mRNA, pre-rRNA, tRNA, small nuclear RNA (snRNA), small nucleolar RNA (snoRNA) and residual ncRNA (Gerstberger et al., 2014) and can alter the fate or function of the bound RNAs during post-transcriptional gene regulation (PTGR), which correlates with the stability, transport, localization, and degradation of different RNAs. They act as important participants in gene regulation (Nishida et al., 2017) and play an important role in maintaining genome integrity (Gerstberger et al., 2014). RBPs have been found to be closely related to many human diseases and to be involved in a wide range of biological processes, such as tumorigenesis, proliferation, development, and apoptosis, by interacting with mRNA (Frisone et al., 2015; Grammatikakis et al., 2017), microRNA (Ciafre and Galardi, 2013), and lncRNA (Luo et al., 2013; Schmitt and Chang, 2016). There are 20,000 proteincoding genes in humans, and $7.5 \%$ of genes are involved in RNA metabolism by binding to RNA (Hentze et al., 2018). But only a few RBPs have received intensive study.

The Cancer Genome Atlas (TCGA) project was launched in 2005 and has accelerated the comprehensive understanding of cancer genomic profiles, thus improving diagnostic methods, therapy standards, and preventive strategies. TCGA has released thousands of high-throughput molecular profiles at different levels, which help researchers better understand cancer pathogenesis, diagnosis, and prognosis. In this study, we integrated the expression profiles of breast cancer at multiple levels (lncRNA, miRNA, and mRNA) across 1,200 samples, including 1079 invasive breast carcinoma samples as well as 104 normal samples. We identified differentially expressed lncRNAs, miRNAs, and mRNAs and then constructing a lncRNA-miRNAmRNA dysregulated network, which is a power-law, small-world network. RBPs were found in the dysregulated network, and some of them are related to overall survival time. In addition, two modules were identified and exhibited a correlation with the overall survival time. Further analysis showed that these modules have significantly different expression patterns in cancer versus normal samples. To better understand these two modules, we mined the literature for the molecules in each module and found that some molecules play important roles in breast cancer biology.

\section{MATERIALS AND METHODS}

\section{RNA-Seq Expression Data Sets and Pre-Processing}

RNA-seq expression data sets of $\sim 1200$ patient samples were downloaded from TCGA (https://portal.gdc.cancer.gov/), comprising 1079 invasive breast carcinoma samples and 104 normal samples (Table S1). MRNA, lncRNA, and miRNA were included in each sample. Using Perl scripts, we combined 1200 files into a single profile. The lncRNA expression profile was extracted from the profile based on the latest annotation from the Ensembl database. The biotypes of known lncRNAs are 3 prime_overlapping_ncrna, ambiguous_orf, antisense, antisense_RNA, lincRNA, ncrna_host, non_coding, non_stop_decay, processed_transcript, retained_intron, sense_intronic, and sense_overlapping. The biotype of proteincoding genes is protein_coding. In total, 19951 mRNA, 15949 
lncRNA, and 1881 miRNA were obtained from TCGA. Based on previously published papers (Yan et al., 2015; Li et al., 2018b; Pan et al., 2018), RNAs with expression 0 in more than $10 \%$ of normal samples were eliminated.

\section{Analysis of Differential Expression Between Breast Carcinoma and Normal Samples}

Differentially expressed molecules were identified through the use of previously reported methods (Li et al., 2015; Li et al., 2018b). Firstly, RNAs were divided into two groups. RNAs with an expression level equal to 0 in $<30 \%$ tumor samples were subjected to a t-test, and RNAs with an expression level equal to 0 in $>30 \%$ tumor samples were subjected to a Fisher's exact test. For the RNAs in the first group, RNAs with a fold change larger than 2 (or smaller than 0.5 ) and an adjusted p-value smaller than 0.01 were identified as differentially expressed. For RNAs in the second group, we determined their expression in binary fashion: ON (expressed, expression value larger than 0) and OFF (not detected, expression value equal 0). Firstly, the frequencies of $\mathrm{ON}$ and OFF in breast carcinoma and normal samples were calculated, respectively. RNAs expressed twice more frequently in cancer than in normal samples were marked as 'ON in cancer'; otherwise, RNAs were marked as 'OFF in Cancer.' Then, for each RNA, the significance of the contingency between ON/OFF and cancer/normal status was calculated by Fisher's exact test with adjustment for multiple testing via the Benjamini-Hochberg method. RNAs with FDR smaller than 0.01 was used. In total, 4269 differentially expressed protein-coding genes were identified, as well as 3057 differentially expressed lncRNAs and 367 differentially expressed miRNAs (Tables S2-S5). Validation of the differentially expressed RNAs was performed by extracting the expression values and normalized them based on Z-score. After that, based on the R package 'pheatmap,' samples were clustered using differentially expressed lncRNAs, miRNAs, and mRNAs, respectively. PCA was also used to cluster the samples.

\section{Ago CLIP-Seq-Supported miRNA Target Sites}

miRNA target sites were predicted using a target prediction algorithm from miRanda (Betel et al., 2010) with the default parameters. 3'UTR was used to predict target sites for mRNA, while for lncRNA, the full length of the lncRNA transcript was used. It has been reported that miRNAs function in the form of ribonucleoprotein complexes, RISCs (RNA-induced silencing complexes) (Fabian et al., 2010), and Argonaute (AGO)-family proteins represent the best-characterized protein components and are central to RISC function (Eulalio et al., 2008; Chekulaeva and Filipowicz, 2009). Ultraviolet (UV) crosslinking and immunoprecipitation (CLIP) was used to identify specific protein-RNA interactions (Konig et al., 2012). Hence the function of the Argonate-RNA-miRNA complex can be verified through CLIP technology (Chou et al., 2013). Here we downloaded AGO 1/2 CLIP-Seq datasets from starBase v2.0 ( $\mathrm{Li}$ et al., 2014a) and identified AGO binding sites to filter candidate miRNA target sites. A target was reserved only if it overlapped with at least one AGO binding site. In total, 41632 miRNAlncRNA regulatory relationships were predicted, including 1176 lncRNAs and 2509 miRNAs, and 1247237 miRNA-mRNA regulatory relationships were predicted, including 18252 protein-coding genes and 2511 miRNAs (Tables S6 and S7).

\section{Constructing the Dysregulated IncRNA- miRNA-mRNA Network}

Based on the interactions of miRNA-lncRNA and miRNAmRNA, we constructed an initial lncRNA-miRNA-mRNA network. A three-step filtering process was then performed: 1) RNAs that were not differentially expressed were filtered; 2) the expression of each RNA pair (miRNA-lncRNA or miRNAmRNA) should be significantly correlated ( $\mathrm{p}$-value $<0.01$ and correlation coefficient $\mid>0.4$ ) across samples based on Pearson correlation; 3) only miRNAs that were shared by both lncRNA and mRNA were considered. The dysregulated network was constructed based on 876 interactions and 539 differentially expressed molecules, including 75 miRNAs, 63 lncRNAs, and 401 protein-coding genes (Table S8). The network was visualized using Cytoscape (Shannon et al., 2003). CytoCluster (Li et al., 2017), a Cytoscape plugin for cluster analysis and visualization of biological networks, was used to identify modules, employing the graphically based IPC-MCE algorithm and adopting the default parameter values ( 0.6 as the Threshold).

\section{Survival Analysis}

The clinical data of all of the breast cancer patients were downloaded from TCGA. Perl scripts were used to extract the information regarding days to last follow up and vital status (alive or dead) for each invasive breast carcinoma patient. For each module, the average value in each sample was used. Modules that relate to the overall survival were identified by clustering the samples into two classes based on K-means Clustering. An R package, 'survival' was then used to 1) construct a surv object using the function 'Surv' based on the status and time, 2) create fitted survival curves with the KaplanMeier algorithm, using the function 'survfit' based on the surv object and class label, and 3) test for a difference between the two survival curves using a log-rank test. P-value $<0.05$ was set as the cutoff. All reported p-values were two-sided.

\section{Functional Enrichment Analysis}

In order to investigate functional roles, GO and KEGG analyses were performed based on protein-coding genes in the network using the Database for Annotation, Visualization, and Integrated Discovery (DAVID, version 6.8) (Huang da et al., 2009; Huang et al., 2009). Cancer hallmarks related GO terms were identified by two previous studies (Subramanian et al., 2005; Plaisier et al., 2012). Additionally, PANTHER (Mi and Thomas, 2009) (https:// reactome.org/) and REACTOME (Croft et al., 2011) (http:// pantherdb.org/) pathway analysis were performed. To further investigate the functional roles, GAD, a database of genetic association data from complex diseases and disorders, was also used by DAVID (Huang da et al., 2009; Huang et al., 2009). 


\section{Cancer Genes}

Two cancer gene lists were used to further validate the roles in cancer. The first one was compiled by by Mertins et al. (2016), who collected 415 oncogenes and tumor suppressors from UniProt (https://www.uniprot.org/) and published papers. Another list of 524 genes that had been implicated in malignant transformation according to a catalog of somatic mutations in cancer (COSMIC, http://cancer.sanger.ac.uk/ cancergenome/projects/census) was collected by Uhlen et al. (Uhlen et al., 2015). In total, 724 potentially cancer-related genes were used (Table S9).

\section{RESULTS}

\section{Differentially Expressed RNAs Distinguish Invasive Breast Carcinoma From Normal Tissues}

We acquired the expression profiles of mRNA, lncRNA, and miRNA from TCGA, which contains 1183 samples, comprising 1079 invasive breast carcinoma samples and 104 normal samples (Table S1). Differentially expressed molecules were identified using the method detailed in Li et al. (2018b). RNAs with an expression level equal to 0 in $<30 \%$ tumor samples were subjected to t-test, and RNAs with an expression level equal to 0 in $>30 \%$ tumor samples were subjected to Fisher's exact test (see Methods). In total, 4269 protein-coding genes that were differentially expressed between invasive breast carcinoma and normal samples were identified, including 2349 up-regulated and 1920 down-regulated genes (Tables S2 and S3). For lncRNAs, 3057 differentially expressed molecules were identified, of which 2033 were up-regulated and 1024 were down-regulated (Tables S2 and S4). Additionally, 367 differentially expressed miRNAs were identified. 152 miRNAs were up-regulated, and 215 were down-regulated (Tables S2 and S5).

We validated our differentially expressed molecules by performing unsupervised hierarchical cluster analyses for the 1179 invasive breast carcinoma samples and 104 normal samples using the $\mathrm{R}$ package 'pheatmap' with the default distance. The invasive breast carcinoma samples were clearly distinguished from normal samples in terms of differentially expressed lncRNAs, protein-coding genes, and miRNAs, respectively (Figures 1A-C). To further check these differentially expressed molecules, principle component analysis (PCA) analyses were performed using the $\mathrm{R}$ function 'prcomp.' Consistent with the unsupervised hierarchical clustering, the first two principal components could distinguish the tumor samples from normal samples (Figures 1D-F).

\section{The Dysregulated Network Is a Biological Network Performing Housekeeping and Cancer-Related Functions}

All of the differentially expressed molecules mentioned above were used to construct the dysregulated network. We predicted miRNA target sites for all protein-coding genes and lncRNAs based on the algorithm from miRanda (Betel et al., 2010), using the default parameters (see Methods). It has been reported that miRNA functions in the form of ribonucleoprotein complexes, RISCs (RNA-induced silencing complexes) (Fabian et al., 2010), and Argonaute (AGO)-family proteins represent the bestcharacterized protein components and are central to RISC function (Eulalio et al., 2008; Chekulaeva and Filipowicz, 2009). Ultraviolet (UV) crosslinking and immunoprecipitation (CLIP) was used to identify specific protein-RNA interactions (Konig et al., 2012). Hence, the function of the Argonate-RNA-miRNA complex can be verified through CLIP technology. Candidate miRNA and target site pairs were filtered by the AGO 1/2 CLIPseq data from starBase (Li et al., 2014a) (see Materials and Methods). A total of 41632 interactions, including 2509 miRNAs and 1176 lncRNA targets, were predicted as well as 1247237 interactions between 2577 miRNAs and 18252 proteincoding genes (Tables S6 and S7). Next, based on the three-step filtering process (see Materials and Methods), a dysregulated network was constructed from 876 interactions and 539 differentially expressed molecules, including 75 miRNAs, 63 lncRNAs, and 401 protein-coding genes (Figure 2A, Table S8).

It has been shown that many biological networks are smallworld networks (Latora and Marchiori, 2001; Wagner and Fell, 2001), which have also been reported to be scale-free networks (Amaral et al., 2000). We tested whether our dysregulated network is a scale-free network by analyzing the degree distribution, which is one of the most important characteristics of a scale-free network and is defined as the number of edges incident to a node. As shown in Figure S1A, more than $88 \%$ of nodes had less than five edges, whereas only $6 \%$ of nodes had more than 10 edges. It fitted a power-law distribution with $R^{2}=$ 0.86 and correlation $=0.99$, suggesting that our network is a scale-free network (Barabasi, 2009). In addition, most of the shortest paths were between 4 and 6 (Figure S1B), which is consistent with the property of a small-world network. Closeness is a measure of how close an individual is to other individuals in a network (Borgatti, 1995; Costenbader and Valente, 2003). The more central a node is, the closer it is to all other nodes. As shown in Figure S1C, the majority of nodes were highly central. Together, these data revealed that our dysregulated network is a scale-free and small-world network, indicating that our network is a canonical biological network.

The functions of the dysregulated network were investigated by using the protein-coding genes in this network to perform functional enrichment analysis (see Methods). All top-ten enriched gene ontology (GO) terms were related to cell cycle, mitotic nuclear division, and nuclear division (Figure 2B). These were all housekeeping functions for maintaining cell survival. We further acquired all of the housekeeping genes identified by Jiang et al. (2018) and found that 84 (21\%) protein-coding genes in our dysregulated network were housekeeping genes (Table S10). In addition, based on a previous study (Salem et al., 2016), we obtained a list of GO terms related to hallmarks of cancer and found that these terms were also enriched in our network (Figure 2C). For example, signal transduction (GO:0007165) and positive regulation of cell proliferation (GO:0008284) are 
A

Differentially expressed IncRNAs

B

Differentially expressed mRNAs

Differentially expressed miRNAs
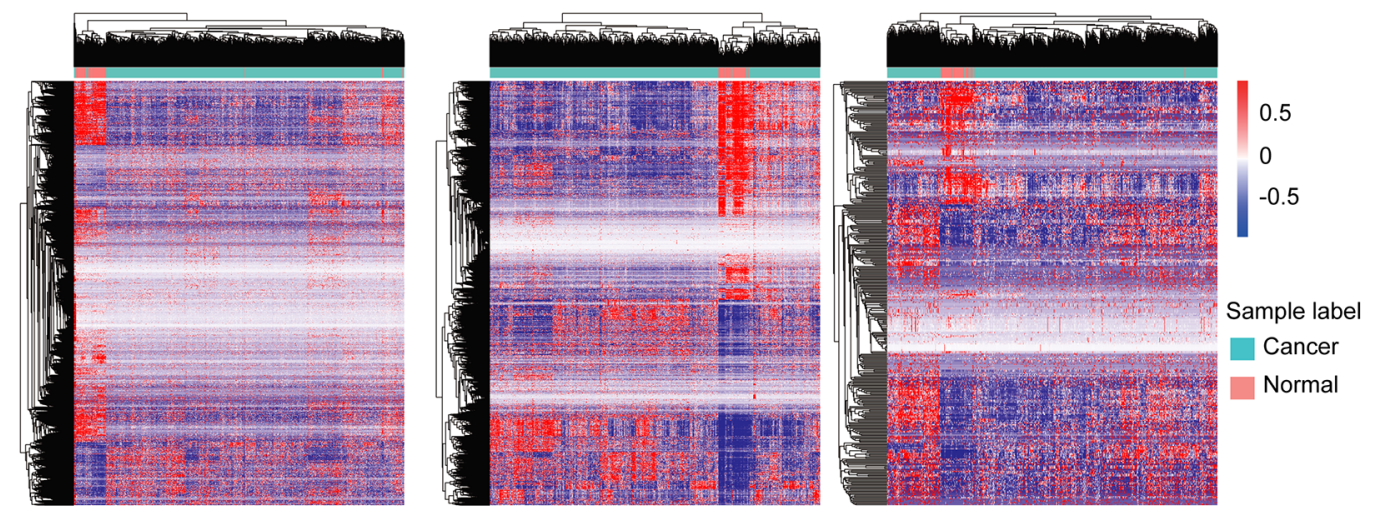

D

E
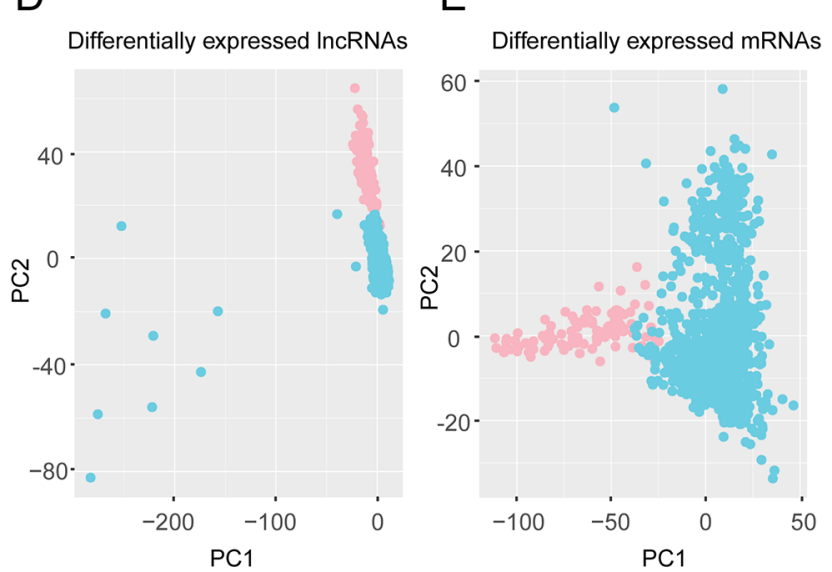

$\mathrm{F}$

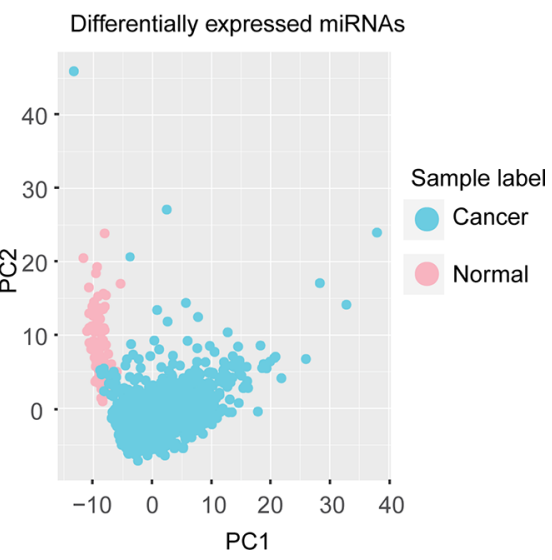

FIGURE 1 | Clustering based on differentially expressed molecules. Unsupervised hierarchical clustering of all samples based on differentially expressed IncRNAs (A) protein-coding genes (B) and miRNAs (C). The unsupervised hierarchical clustering was performed using an R package, 'pheatmap' with the default distance setting, Euclidean distance. (D-F) PCA analysis based on differentially expressed IncRNAs (D), protein-coding genes (E), and miRNAs (F). PCA analysis was performed by the R function 'prcomp'.

Self Sufficiency in Growth Signals, while negative regulation of cell proliferation (GO:0008285) and negative regulation of cell cycle (GO:0045786) are Insensitivity to Antigrowth Signals. Taken together, our dysregulated network demonstrated important and functional roles.

Moreover, we performed pathway enrichment analyses using three different pathway databases, the Kyoto Encyclopedia of Genes and Genomes (KEGG), PANTHER (Mi and Thomas, 2009), and REACTOME (Croft et al., 2011). For the KEGG pathway, housekeeping and cancer-related functions were again enriched (Figure S2A). The housekeeping functions were cell cycle and axon guidance, and the cancer-related functions were pathways in cancer, Melanoma, Colorectal cancer and Prostate cancer (Figure S2A). For the PANTHER and REACTOME pathway databases, most of the top terms were housekeeping functions (Figures S2B, C).

To further validate their important roles in cancer, we obtained 415 oncogenes and tumor suppressors from Mertins et al. (2016) and 524 genes that have been implicated in malignant transformation from Uhlen et al. (2015). In total, 724 cancer genes were used (Table S9). 656 of them were expressed in our dataset, and 31 were in our network (Figure S2D). Based on a hypergeometric test, the p-value was 7.46E-05, which suggested that our dysregulated network was significantly enriched in cancer-related genes. We further performed functional enrichment analysis using DAVID (Huang da et al., 2009; Huang et al., 2009) based on the Genetic Association Database (GAD), which is a database of genetic association data from complex diseases and disorders. Surprisingly, breast cancer was the most enriched term (Figure 2D), which corroborated the important roles of our dysregulated network in cancer biology.

\section{RBPs in Our Dysregulated Network}

Next, we investigated the RBPs in our dysregulated network. Based on published papers (Cook et al., 2011; Gerstberger et al., 2014; Fredericks et al., 2015; Hentze et al., 2018), 58 RBPs were found in our dysregulated network, of which 28 were upregulated and 30 were downregulated (Table S11). To improve our 


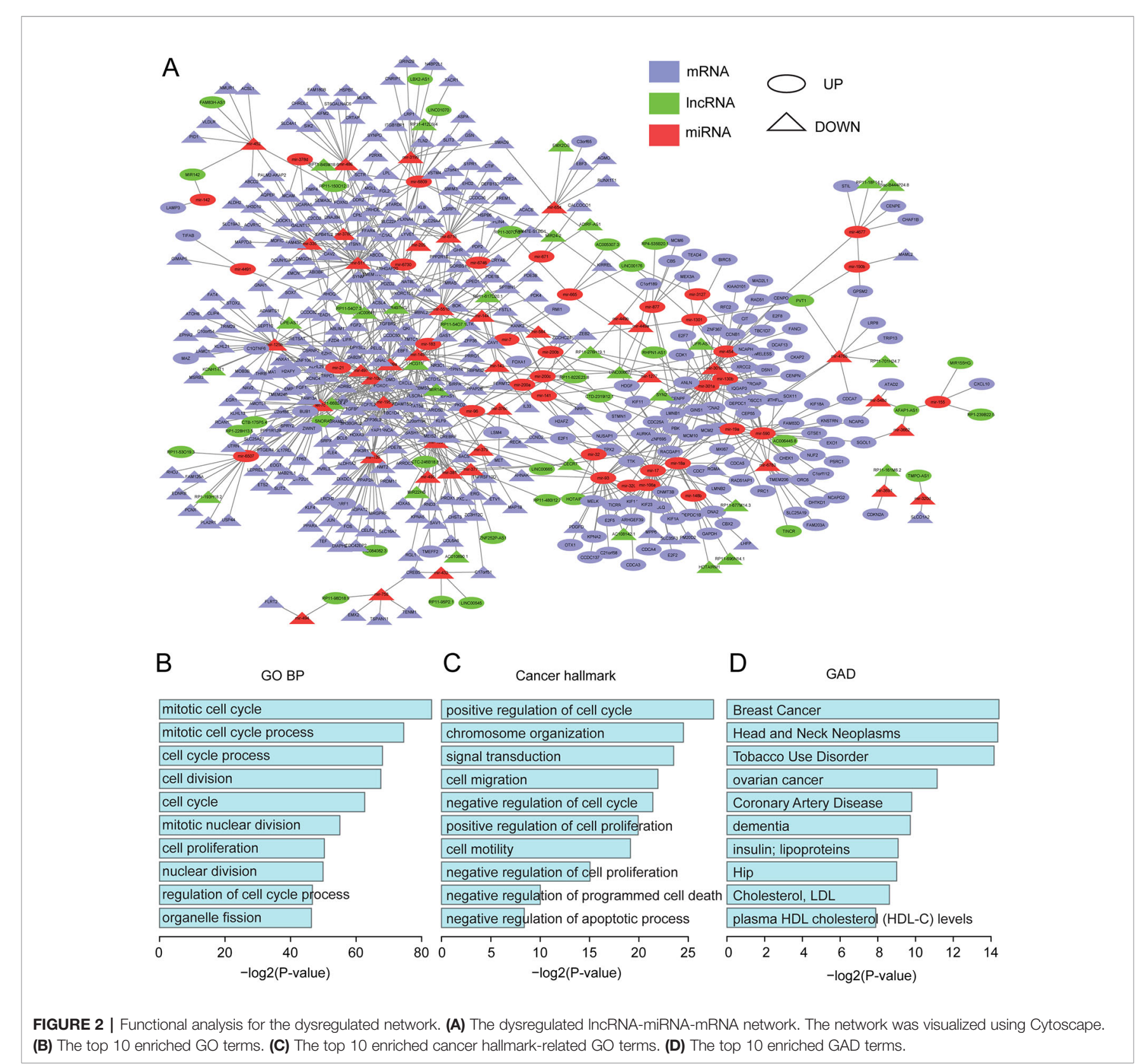

(B) The top 10 enriched GO terms. (C) The top 10 enriched cancer hallmark-related GO terms. (D) The top 10 enriched GAD terms.

understanding of the roles of RBP in invasive breast carcinoma, STRING (https://string-db.org/) was used to construct a proteinprotein interaction (PPI) network (Figure 3A). Random networks of the same size were generated by STRING, which was used to assess whether the given network had more internal interactions than would be expected for a random set of the same size. A small PPI enrichment $\mathrm{p}$-value indicates that the nodes are not random and that the observed number of edges is significant. Based on STRING, the PPI enrichment p-value was $1.0 \mathrm{e}-16$, which means that these RBPs have more interactions than would occur in a random set. This enrichment indicated that these RBPs are at least partially biologically connected as a group. The GO analysis showed that all top 10 molecular function (MF) terms were binding-related functions and the top two were poly(A) RNA binding and RNA binding, which further confirms that they are RBPs (Figure 3B), and that these RBPs are involved biological processes that are essential to maintain cell survival like cell cycle, cell division, DNA packaging, and chromosome organization (Figure 3C). Moreover, GAD enrichment analysis was also performed, and it is worth noting that breast cancer was again the most enriched term (Figure S3).

To investigate whether these RBPs were associated with prognosis in invasive breast carcinoma patients, the overall survival for each RBP was calculated using the $\mathrm{R}$ package 'survival' (see Methods). Ten RBPs (CDKN2A, DCAF13, DNMT3B, EXO1, FANCI, KPNA2, RACGAP1, SORBS1, TP63, and ZNF106) were significantly associated with overall survival, including seven upregulated and three downregulated 


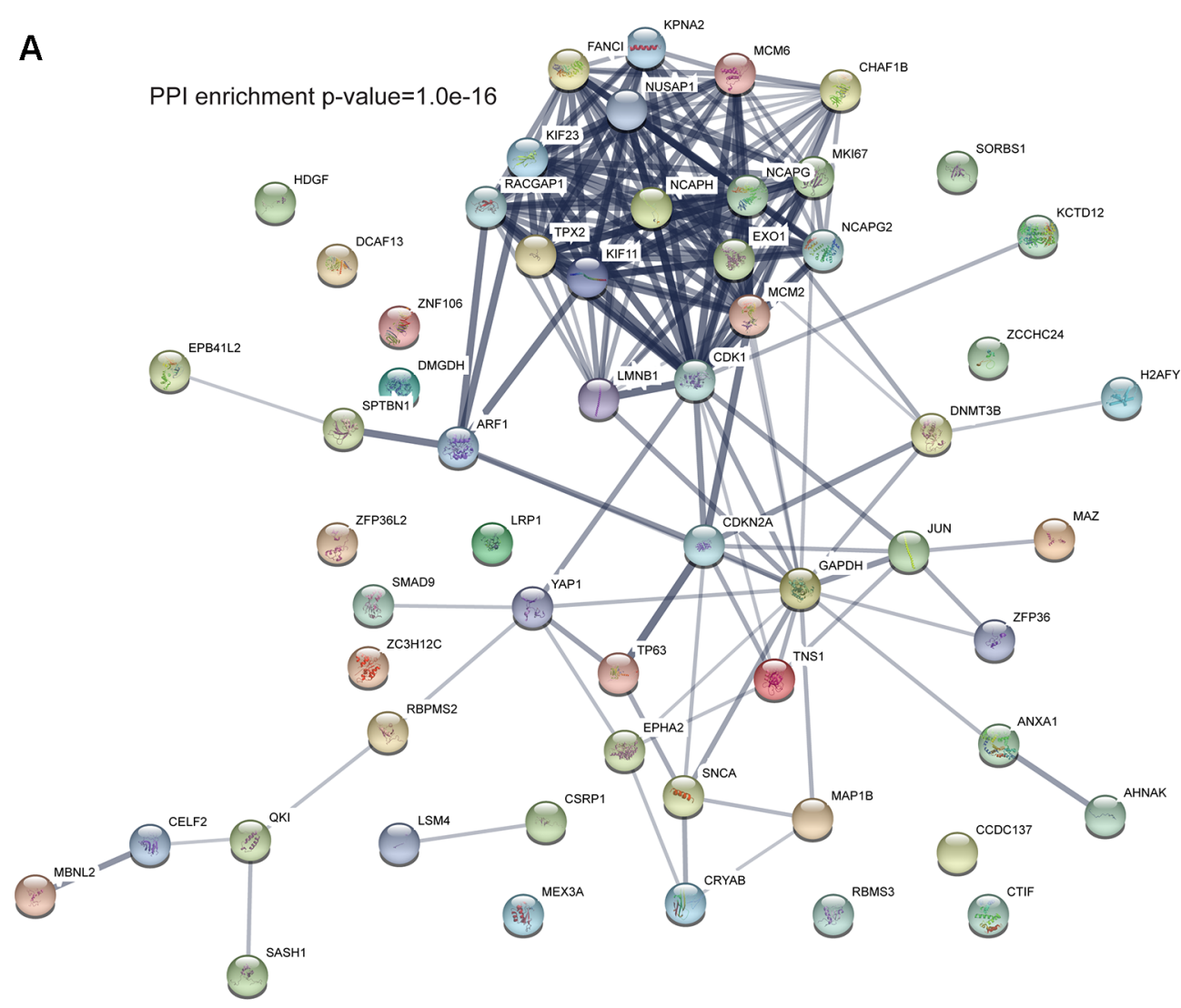

B

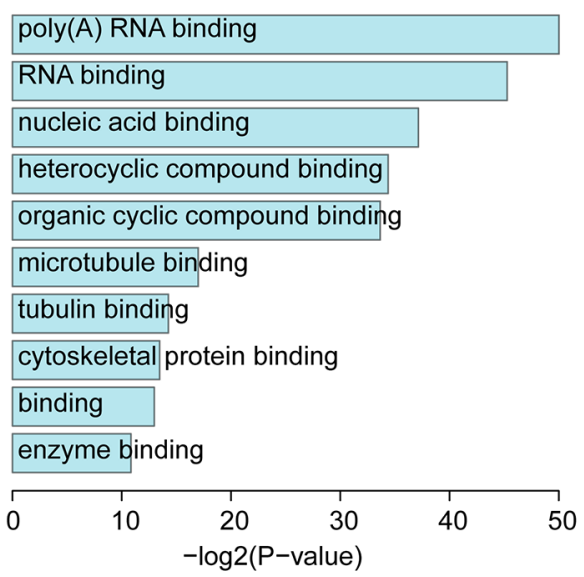

C

GO BP

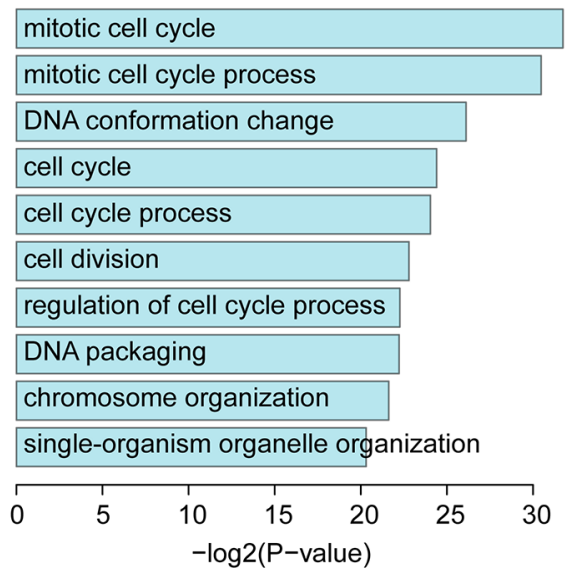

FIGURE 3 | RBPs in the dysregulated network. (A) Protein-protein interaction (PPI) network of RBPs based on STRING. (B) The top 10 enriched GO MF terms. (C) The top 10 enriched GO BP terms. BP, biological process.

RBPs (Figures S4 and S5). Notably, some were reported to play roles in breast cancer (see Discussion). Overexpression of DCAF13, DNMT3B, KPNA2, EXO1, FANCI, RACGAP1, and ZNF106 in invasive breast carcinoma patients showed poor survival, while overexpression of CDKN2A, SORBS1, and TP63 showed better survival (Figures S4 and S5).

\section{Modules in the Dysregulated Network Relate to the Survival of Invasive Breast Carcinoma Patients}

To further investigate the roles of our dysregulated network, CytoCluster (Li et al., 2017), a Cytoscape plugin for cluster analysis and visualization of biological networks, was used to 
identify modules (see Methods). Subsequently, to explore the relationship between the modules and the prognosis of patients with invasive breast carcinoma, the overall survival for each module in invasive breast carcinoma patients was investigated (see Methods). We found that two modules were significantly $(\mathrm{p}<0.05)$ correlated with overall survival (Figures 4A-D). Moreover, their expression patterns in normal and invasive breast carcinoma samples were assessed. These two modules
A

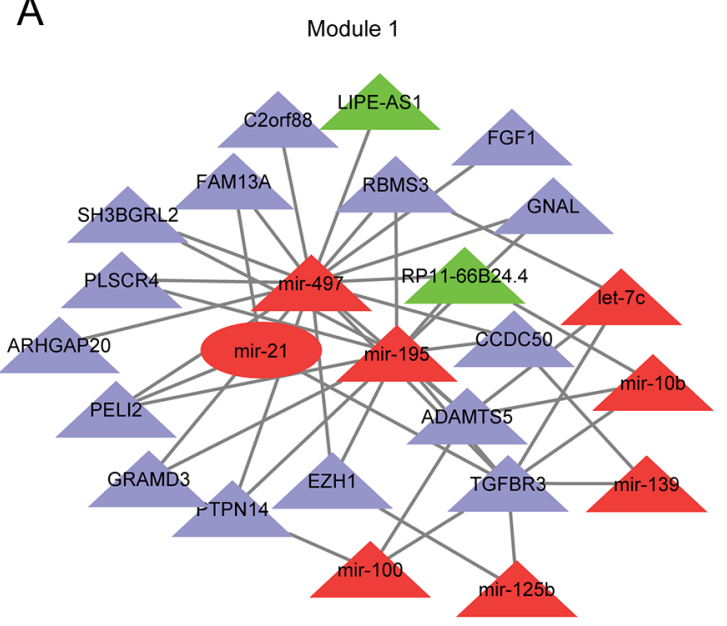

C
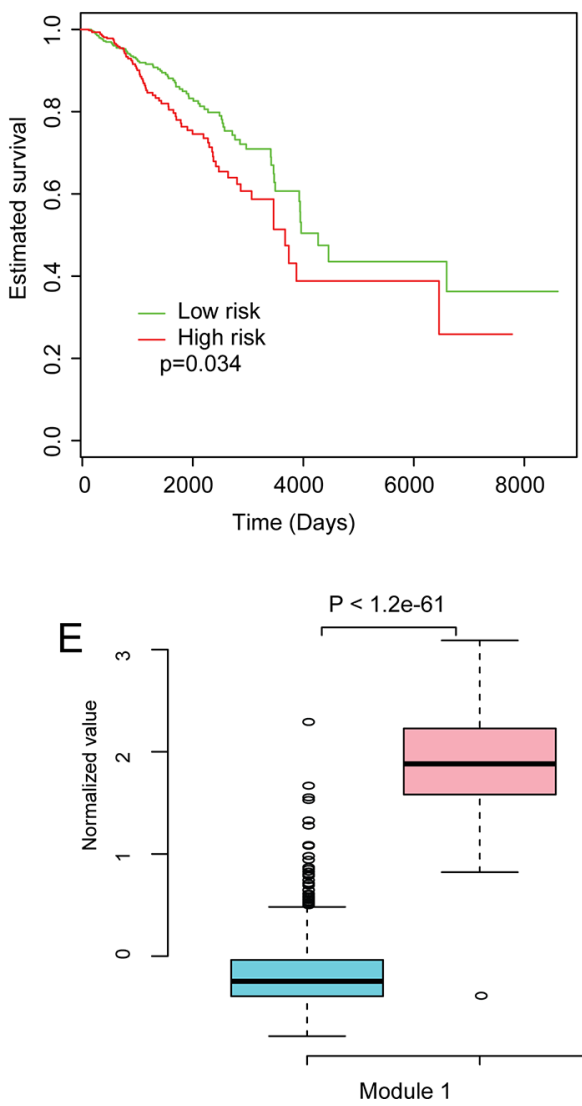

B

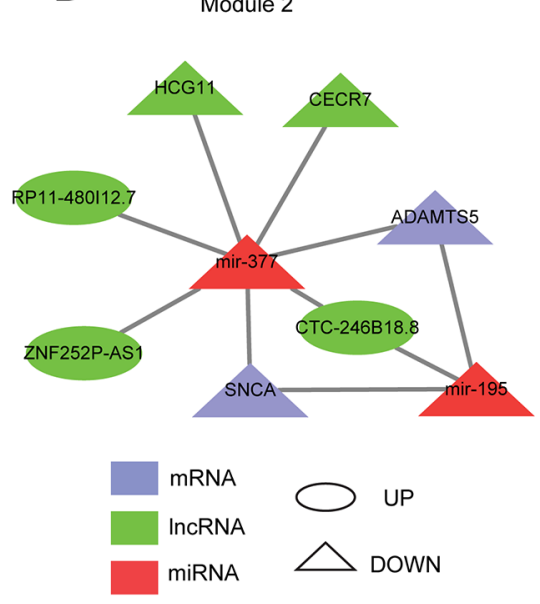

D

Module 2
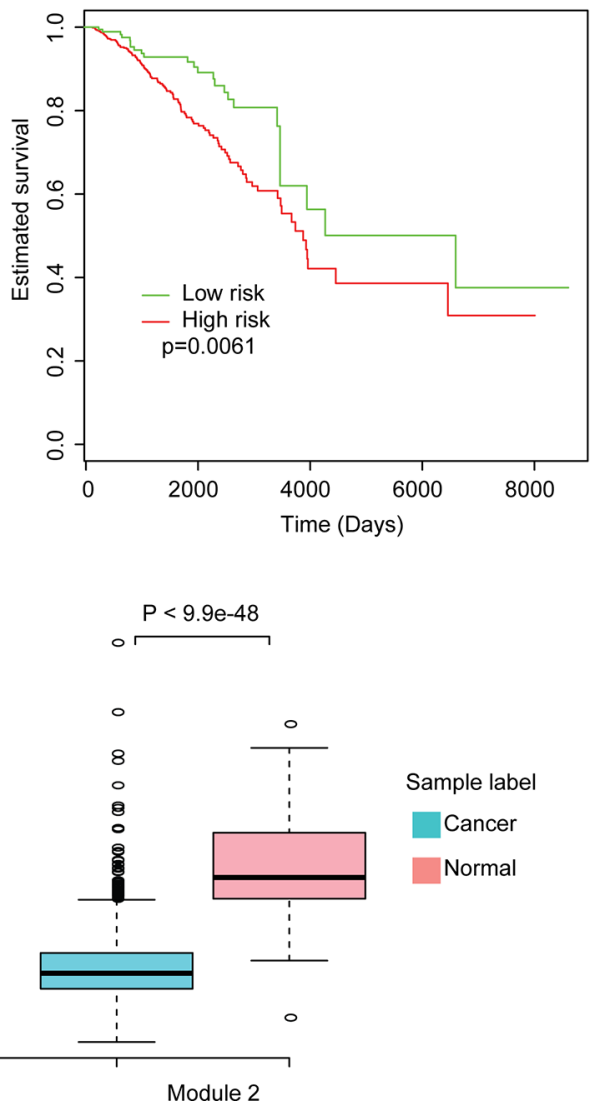

FIGURE 4 | Analysis of modules identified from the dysregulated network. (A, B) The two modules identified from the dysregulated network using Cytoscape with default parameters. (C, D) Kaplan-Meier plot of survival for these two modules. (E) Expression patterns of the modules in normal and cancer samples. The average expression value of each molecule crossing all normal/cancer samples was used. 
showed significant differences in expression patterns between normal and invasive breast carcinoma samples (Figure 4E). Both showed significantly lower expression in invasive breast carcinoma samples, indicating that lower expression of these modules contributes to the development of invasive breast carcinoma.

In addition, to further investigate the functions of these two modules in breast cancer, literature-mining was used for the molecules in each module. Module 1 had 25 nodes, including eight miRNAs, two lncRNAs, and 15 protein-coding genes. Twenty-two of the molecules, including all of the miRNAs, have been shown to play important roles in breast cancer. For example, mir-195 inhibits tumor growth and metastasis in breast cancer cells (Singh et al., 2015; Wang et al., 2016c). Mir-497 contributes to cell proliferation, migration, and invasion of estrogen receptor alpha-negative breast cancer by targeting estrogen-related receptor alpha (Han et al., 2016; Wu et al., 2016b). TGFBR3 inhibits breast cancer progression through TGF-beta signaling (Lee et al., 2010). In addition, other molecules in module 1 such as ADAMTS5 (Fontanil et al., 2017), ARHGAP20 (Asaduzzaman et al., 2017), C2orf88 (Lo et al., 2015), EZH1(Liu et al., 2012), FAM13A (Goto-Yamaguchi et al., 2018), FGF1 (Slattery et al., 2013), GNAL (Yi et al., 2009), GRAMD3 (Boiles et al., 2015), PELI2 (Zang et al., 2017), PLSCR4 (Sahay et al., 2015), PTPN14 (Belle et al., 2015), RBMS3 (Zhu et al., 2019a), SH3BGRL2 (Alexe et al., 2007; Wen et al., 2018), let-7c (Fu et al., 2017), mir-100 (Jiang et al., 2016b), mir-10b (Wang et al., 2016b), mir-125b (Wang et al., 2019a), mir-139 (Dai et al., 2017), and mir-21 (Yan et al., 2008; Yanwirasti and Arisanty, 2017; Zhu et al., 2019b) have been reported to play important roles in breast cancer. Module 2 had nine nodes, including two miRNAs, five lncRNAs, and two protein-coding genes. Five of these molecules have been shown to play important roles in breast cancer. For example, Wang et al., (2019b) reported that overexpression of miR-377 correlates with better prognosis in triple-negative breast cancer. ADAMTS-5 may alter the cellular microenvironment, affecting the balance between protumor and antitumor effects (Fontanil et al., 2017). SNCA is the hub gene and is involved in promoting tumor invasion in breast cancer (Serra-Musach et al., 2012; Dang et al., 2016). Besides, molecules in module 2 like the lncRNAs (HCG11) (Liu et al., 2016) and mir-195 (Singh et al., 2015; Wang et al., 2016c) have also been reported to play roles in breast cancer. We also performed key driver analysis (KDA) (Bin Zhang, 2013) to identify key drivers in our network, and all of the miRNAs from our two modules were identified as key drivers. All of these results imply the important roles and vital functions of these two modules in breast cancer biology.

\section{DISCUSSION}

Breast cancer is a leading type of cancer in women worldwide (Stewart and Wild, 2014). Many improvements have been made in diagnostic techniques, surgical skills, and medical treatments relating to breast cancer in the past decades. However, it still caused 522,000 deaths in 2012 (Stewart and Wild, 2014). It is imperative to improve the diagnosis and treatment of breast cancer further. Therefore, the identification of cancer-related molecules and the exact regulatory mechanism of breast cancer initiation and development are attracting increasing attention.

It has been reported that lncRNAs and miRNAs play important roles in breast cancer, as do protein-coding genes (Cizkova et al., 2013; Li et al., 2014b; Kim et al., 2015; Yang et al., 2018b). Here we integrated the expression data of lncRNA, miRNA, and protein-coding genes based on $\sim 1200$ invasive breast carcinoma and normal samples from TCGA. A total of 4269 differentially expressed protein-coding genes, 3057 differentially expressed lncRNAs, and 367 differentially expressed miRNAs were identified. Based on unsupervised hierarchical clustering and PCA, the samples from invasive breast cancer were distinguished from the normal samples. To construct a dysregulated network, we predicted miRNA targets using an algorithm from miRanda (Betel et al., 2010) with the default parameters. As mature miRNA is part of an active RNAinduced silencing complex (RISC) (Rana, 2007) and the Ago family is central to RISC function (Tang, 2005), AGO CLIP-Seq data were applied to achieve highly convincing miRNA targets. Based on the differentially expressed lncRNAs, miRNAs, and protein-coding genes, an initial dysregulated lncRNA-miRNAmRNA network was built. After three-step filtering, the final network was constructed, consisting of 876 interactions and 539 differentially expressed molecules.

Next, we analyzed this network through different aspectsthe distribution of degree, shortest path, and closeness centrality - which showed that the dysregulated network is a scale-free, small-world network and a meaningful biological network. To further understand the function of the dysregulated network, functional enrichment analysis was performed. The top-10 GO terms showed housekeeping functions in our network. Furthermore, terms related to cancer hallmarks were also found, based on a previous study (Salem et al., 2016). Enrichment analysis with three different pathway databases supported the housekeeping and cancer-related functions in our dysregulated network. Based on two previous studies, 716 potential cancer genes were obtained, and further analysis showed enrichment in these cancer-related genes. Furthermore, we found that breast cancer was the most enriched term based on GAD, suggesting the important role of our dysregulated network in cancer biology.

It was known that RBPs play a central role in the regulation of gene expression, and dysregulated expression of RBPs has been related to the development of cancers (Galante et al., 2009; Bebee et al., 2014; Wang et al., 2015; Correa et al., 2016). In the present study, we identified 58 RBPs in our dysregulated network, and these were confirmed by GO BP analysis. These RBPs are involved in biological processes that are essential to maintaining cell survival. Based on STRING, we found that these RBPs had more interactions among themselves than what would be expected, indicating that they are at least partially biologically connected. Interestingly, GAD enrichment analysis again showed that breast cancer was the most enriched 
term. In addition, 10 RBPs were found to be associated with the overall survival of invasive breast carcinoma patients, which suggested that they might be associated with tumor progression, invasion, and aggressiveness. Indeed, some have been reported to play roles in breast cancer. TP63 is a sequencespecific DNA binding transcriptional activator or repressor (Zhou et al., 2016b). In breast cancer, high expression of TP63 coupled with STAT6 has been shown to be associated with longer metastasis-free survival, indicating that TP63 could be involved in inhibiting the migration of breast cancer cells (Papageorgis et al., 2015). By silencing TP63 expression, breast cancer cells acquired increasing resistance to cisplatin, suggesting its role in drug reaction (Mendoza-Rodriguez et al., 2019). SORBS1 is an adaptor protein, and its overexpression inhibits the invasive capacity of tumor cells in breast cancer patients. Silencing SORBS1 promoted EMT and weakened chemotherapy sensitivity (Song et al., 2017). DCAF13, located in chromosome 8q22.3, has been shown to be amplified in breast cancer. Overexpression of DCAF13 was associated with worse prognosis and might be involved in regulating cell cycle progression (Chin et al., 2007; Cao et al., 2017). By targeting DNMT3b, miR-221 became involved in tumorigenicity through regulating the stemness of breast cancer cells (Roscigno et al., 2016). Additionally, DNMT3B helped maintain the CAF function of promoting breast cancer malignance (Tang et al., 2019). RBPs are important in tumor development, and their role still needs to be explored more.

Using CytoCluster (Li et al., 2017), we identified modules that were significantly related to the overall survival time. These two modules had significantly different expression patterns in cancer and in normal samples. Moreover, the literature mining revealed that some molecules in each module play important roles in breast cancer. In module 1 , there were 25 nodes, including eight miRNAs, two lncRNAs, and 15 mRNAs. It had been reported that the upregulation of mir-497 inhibited cell proliferation, migration, and invasion in breast cancer (Han et al., 2016; Wang et al., 2016a; Wu et al., 2016b) and that mir-195 inhibited tumor growth, invasion, and metastasis by targeting other RNAs in breast cancer (Singh et al., 2015; Wang et al., 2016c). Importantly, mir-497 and mir-195 were the hub nodes in this module, indicating their essential role in the module. However, these two miRNAs were down-regulated in breast cancer, which means that the inhibition was lost, contributing to the development of breast cancer. Consistent with the tumorsuppressive role of these two miRNAs, TGFBR3 was reported to suppress breast cancer progression through TGF-beta signaling (Lee et al., 2010), and RBMS3 and PTPN14 were also shown to play roles in inhibiting metastasis (Belle et al., 2015; Zhu et al., 2019a). These data imply that the function of module 1 may be to inhibit cancer progress and metastasis and that these functional miRNAs may affect breast cancer through TGFBR3, RBMS3, and PTPN14. Additionally, LIPE-AS1 (lncRNA), RP11-66B24.4 (lncRNA), and CCDC50 (mRNA) have not been reported in BRCA, but LIPE-AS1 interacted with miRNA-497 and slightly correlated with overall survival $(\mathrm{p}=0.075)$ and both RP1166B24.4 and CCDC50 are regulated by the two hub miRNAs, which suggested that they might act as main or auxiliary regulators in the progression and metastasis of BRCA. In module 2, mRNA ADAMTS5 was reported to play roles during migration and invasion in breast cancer (Fontanil et al., 2017). It also functions as a tumor suppressor by inhibiting migration, invasion, and angiogenesis in human gastric cancer (Huang et al., 2019). Besides, two other studies have shown that the upregulation of ADAMTS5 promotes progression in colorectal cancer and drives metastasis in colon and non-small cell lung cancer (Gu et al., 2016; Yu et al., 2016). Another mRNA, SNCA, was also reported to be involved in tumor development by inhibiting invasion and inducing apoptosis (Li et al., 2018c; Yan et al., 2018). Thus, the function of module 2 might relate to cancer progression and survival. Previous studies have shown that miRNAs may function as tumor suppressors or oncogenes in tumor development, invasion, and metastasis. In module 2, mir-377 is the hub node and may be the core molecule involved in breast cancer due to its interactions with other molecules. Moreover, mir-377 has been reported to inhibit proliferation and metastasis in gastric cancer and pancreatic cancer (Chang et al., 2016; Wang et al., 2017). mir-195 was also important in BRCA, as it was shown that mir-195 could inhibit the invasion and metastasis of breast cancer (Singh et al., 2015; Wang et al., 2016c). IncRNA CECR7 interacts with mir-377 and had been reported to play a role in hepatocellular carcinoma (Zhang et al., 2015). In addition to the molecules reported to play roles in breast cancer, some novel candidate biomarkers, which may also be important to breast cancer, were found, but more evidence is needed in future.

Many studies have performed integrative analyses of TCGA breast cancer data through networks. For example, Yin et al. (2016) focused on identifying miRNA-mRNA pairs and constructed a miRNA target network in invasive breast carcinoma. Li et al. (2018a) found that some of the correlations between microRNA and target genes declined in cancer compared to normal across multiple cancers. Wu et al. (2016a) found two kinds of lncRNA-mRNA co-expression patterns: 1) correlations between lncRNA-mRNA in cancer were reversed compared to normal; 2) correlations between lncRNA-mRNA in cancer were similar to normal. Xiao et al. (2018) compared the differential genes between ER+ and ERand constructed a ceRNA network and found that some molecules correlated with prognosis. Yang et al. (2018a) compared the differentially expressed genes in Triple-Negative Breast Cancer and also constructed a ceRNA network. Some molecules correlated with prognosis were identified and validated by qRT-PCR. Sun et al. (2019) identified eight lncRNAs as the prognosis signature for breast cancer using a ceRNA and WGCNA network. Gao et al. (2019) built a ceRNA and found some prognosis-related molecules (four lncRNAs, two miRNAs, and two mRNAs). Most studies built a ceRNA network, which contains molecules that are not differentially expressed. However, the integrated dysregulated network in this study consists of differentially expressed lncRNAs, miRNAs, and mRNAs only, and we identified RBPs and modules that can stratify patients into high- and low-risk subgroups. Moreover, 
each module not only relates to prognosis but also contains RNAs that have been reported to play roles in breast cancer.

It is well known that the expression of non-coding RNAs is highly tissue- and cell-type specific, providing important clues about their specific functions in response to contextual demands (Mercer et al., 2008; Cabili et al., 2011; Jiang et al., 2016a). Here, we identified patient survival-associated modules including noncoding RNAs in invasive breast carcinoma, and this interpretation was supported in many ways. All molecules in the modules were differentially expressed in invasive breast carcinoma, indicating the potential roles of these molecules. The modules came from a scale-free biological network that performs functions that are related to housekeeping and are cancer hallmarks. More importantly, these two modules were significantly correlated with overall survival. Moreover, many papers have shown clues that molecules in our networks play roles in the progression of breast cancer, and KDA analysis also showed that the molecules in our networks are key drivers. Based on these strands of evidence, our results are credible. However, there are limitations to this study. Firstly, it is a network-based study. Secondly, our study is only based on bioinformatics analysis. Experiments are needed to support the identifications of functional roles.

\section{CONCLUSIONS}

In summary, using a network-based strategy, we provided a framework integrating miRNAs, mRNAs, and lncRNAs that are differentially expressed in breast cancer to identify biomarkers. Although further validation is still needed to support the potential roles of the RBPs and two modules,

\section{REFERENCES}

Alexe, G., Dalgin, G. S., Ganesan, S., Delisi, C., and Bhanot, G. (2007). Analysis of breast cancer progression using principal component analysis and clustering. J. Biosci. 32 (5), 1027-1039. doi: 10.1007/s12038-007-0102-4

Amaral, L. A. N., Scala, A., Barthelemy, M., and Stanley, H. E. (2000). Classes of small-world networks. Proc. Natl. Acad. Sci. U. S. A. 97 (21), 11149-11152. doi: $10.1073 /$ pnas.200327197

Asaduzzaman, M., Constantinou, S., Min, H., Gallon, J., Lin, M. L., Singh, P., et al. (2017). Tumour suppressor EP300, a modulator of paclitaxel resistance and stemness, is downregulated in metaplastic breast cancer. Breast Cancer Res. Treat 163 (3), 461-474. doi: 10.1007/s10549-017-4202-z

Barabasi, A. L. (2009). Scale-free networks: a decade and beyond. Science 325 (5939), 412-413. doi: 10.1126/science.1173299

Bebee, T. W., Cieply, B. W., and Carstens, R. P. (2014). Genome-wide activities of RNA binding proteins that regulate cellular changes in the Epithelial to Mesenchymal Transition (EMT). Syst. Biol. RNA Binding Proteins 825, 267-302. doi: 10.1007/ 978-1-4939-1221-6

Belle, L., Ali, N., Lonic, A., Li, X., Paltridge, J. L., Roslan, S., et al. (2015). The tyrosine phosphatase PTPN14 (Pez) inhibits metastasis by altering protein trafficking. Sci. Signal 8 (364), ra18. doi: 10.1126/scisignal.2005547

Betel, D., Koppal, A., Agius, P., Sander, C., and Leslie, C. (2010). Comprehensive modeling of microRNA targets predicts functional non-conserved and noncanonical sites. Genome Biol. 11 (8), R90. doi: 10.1186/gb-2010-11-8-r90

Bin Zhang, J. Z. (2013). Identification of key causal regulators in gene networks by Bin Zhang and Jun Zhu. Proceedings of the World Congress on Engineering II. many strands of evidence show the correlations between our two modules and breast cancer. Overall, our dysregulated network provides new insights into outcome prediction for invasive breast cancers.

\section{DATA AVAILABILITY STATEMENT}

Publicly available datasets were analyzed in this study. This data can be found here: https://portal.gdc.cancer.gov/.

\section{AUTHOR CONTRIBUTIONS}

CJ and YD were responsible for the statistical analysis, contributed to the acquisition of data, and were major contributors to writing the manuscript. QS and YX assisted with data analysis and revised the manuscript. All authors read and approved the final manuscript.

\section{ACKNOWLEDGMENTS}

We sincerely thank the public database: TCGA.

\section{SUPPLEMENTARY MATERIAL}

The Supplementary Material for this article can be found online at: https://www.frontiersin.org/articles/10.3389/fgene.2019. 01284/full\#supplementary-material

Boiles, A. R., Prasanna, D., Shaker, A. B., Oswald, M., Mason, C., Keogh, M., et al (2015). Integrated analysis of miRNAs expression in breast cancer patients to detect genes deregulation involved in malignant transformation. J. Clin. Oncol. 33 (15). doi: 10.1200/jco.2015.33.15_suppl.e12560

Borgatti, S. P. (1995). Centrality and AIDS. Connections 18 (1), 112-114.

Cabili, M. N., Trapnell, C., Goff, L., Koziol, M., Tazon-Vega, B., Regev, A., et al. (2011). Integrative annotation of human large intergenic noncoding RNAs reveals global properties and specific subclasses. Genes Dev. 25 (18), 1915-1927. doi: 10.1101/ gad. 17446611

Calin, G. A., and Croce, C. M. (2006). MicroRNA signatures in human cancers. Nat. Rev. Cancer 6 (11), 857-866. doi: 10.1038/nrc1997

Cao, J. Z., Hou, P. J., Chen, J. M., Wang, P. H., Wang, W. Q., Liu, W., et al. (2017). The overexpression and prognostic role of DCAF13 in hepatocellular carcinoma. Tumor Biol. 39 (6), 1010428317705753. doi: 10.1177/1010428317705753

Chang, W., Liu, M., Xu, J., Fu, H., Zhou, B., Yuan, T., et al. (2016). MiR-377 inhibits the proliferation of pancreatic cancer by targeting Pim-3. Tumour Biol. 37 (11), 14813-14824. doi: 10.1007/s13277-016-5295-4

Chekulaeva, M., and Filipowicz, W. (2009). Mechanisms of miRNA-mediated post-transcriptional regulation in animal cells. Curr. Opin. Cell Biol. 21 (3), 452-460. doi: 10.1016/j.ceb.2009.04.009

Chin, S. F., Teschendorff, A. E., Marioni, J. C., Wang, Y., Barbosa-Morais, N. L., Thorne, N. P., et al. (2007). High-resolution aCGH and expression profiling identifies a novel genomic subtype of ER negative breast cancer. Genome Biol. 8 (10), R215. doi: 10.1186/gb-2007-8-10-r215

Chou, C. H., Lin, F. M., Chou, M. T., Hsu, S. D., Chang, T. H., Weng, S. L., et al. (2013). A computational approach for identifying microRNA-target 
interactions using high-throughput CLIP and PAR-CLIP sequencing. BMC Genomics 14 (Suppl 1), S2. doi: 10.1186/1471-2164-14-S1-S2

Ciafre, S. A., and Galardi, S. (2013). microRNAs and RNA-binding proteins A complex network of interactions and reciprocal regulations in cancer. RNA Biol. 10 (6), 935-943. doi: 10.4161/rna.24641

Cizkova, M., Vacher, S., Meseure, D., Trassard, M., Susini, A., Mlcuchova, D., et al. (2013). PIK3R1 underexpression is an independent prognostic marker in breast cancer. BMC Cancer 13. doi: 10.1186/1471-2407-13-545

Cook, K. B., Kazan, H., Zuberi, K., Morris, Q., and Hughes, T. R. (2011). RBPDB: a database of RNA-binding specificities. Nucleic Acids Res. 39, D301-D308. doi: 10.1093/nar/gkq1069

Correa, B. R., de Araujo, P. R., Qiao, M., Burns, S. C., Chen, C., Schlegel, R., et al. (2016). Functional genomics analyses of RNA-binding proteins reveal the splicing regulator SNRPB as an oncogenic candidate in glioblastoma. Genome Biol. 17, 125. doi: 10.1186/s13059-016-0990-4

Costenbader, E., and Valente, T. W. (2003). The stability of centrality measures when networks are sampled. Soc. Networks 25 (4), 283-307. doi: 10.1016/ S0378-8733(03)00012-1

Croft, D., O’Kelly, G., Wu, G., Haw, R., Gillespie, M., Matthews, L., et al. (2011). Reactome: a database of reactions, pathways and biological processes. Nucleic Acids Res. 39 (Database issue), D691-D697. doi: 10.1093/nar/gkq1018

Dai, H., Gallagher, D., Schmitt, S., Pessetto, Z. Y., Fan, F., Godwin, A. K., et al. (2017). Role of miR-139 as a surrogate marker for tumor aggression in breast cancer. Hum. Pathol. 61, 68-77. doi: 10.1016/j.humpath.2016.11.001

Dang, T. T., Westcott, J. M., Maine, E. A., Kanchwala, M., Xing, C., and Pearson, G. W. (2016). DeltaNp63alpha induces the expression of FAT2 and Slug to promote tumor invasion. Oncotarget 7 (19), 28592-28611. doi: 10.18632/oncotarget8696

Eulalio, A., Huntzinger, E., and Izaurralde, E. (2008). Getting to the root of miRNA-mediated gene silencing. Cell 132 (1), 9-14. doi: 10.1016/ j.cell.2007.12.024

Fabian, M. R., Sonenberg, N., and Filipowicz, W. (2010). Regulation of mRNA translation and stability by microRNAs. Annu. Rev. Biochem. 79, 351-379. doi: 10.1146/annurev-biochem-060308-103103

Fontanil, T., Alvarez-Teijeiro, S., Villaronga, M. A., Mohamedi, Y., Solares, L., Moncada-Pazos, A., et al. (2017). Cleavage of Fibulin-2 by the aggrecanases ADAMTS- 4 and ADAMTS- 5 contributes to the tumorigenic potential of breast cancer cells. Oncotarget 8 (8), 13716-13729. doi: 10.18632/ oncotarget.14627

Fredericks, A. M., Cygan, K. J., Brown, B. A., and Fairbrother, W. G. (2015). RNAbinding proteins: splicing factors and disease. Biomolecules 5 (2), 893-909. doi: 10.3390/biom5020893

Frisone, P., Pradella, D., Di Matteo, A., Belloni, E., Ghigna, C., and Paronetto, M. P. (2015). SAM68: signal transduction and RNA metabolism in human cancer. BioMed. Res. Int. 2015, 528954. doi: 10.1155/2015/528954

Fu, X., Mao, X., Wang, Y., Ding, X., and Li, Y. (2017). Let-7c-5p inhibits cell proliferation and induces cell apoptosis by targeting ERCC6 in breast cancer. Oncol. Rep. 38 (3), 1851-1856. doi: 10.3892/or.20175839

Galante, P. A. F., Sandhu, D., Abreu, R. D., Gradassi, M., Slager, N., Vogel, C., et al. (2009). A comprehensive in silico expression analysis of RNA binding proteins in normal and tumor tissue Identification of potential players in tumor formation. RNA Biol. 6 (4), 426-433. doi: 10.4161/rna.6.4.8841

Gao, C., Li, H., Zhuang, J., Zhang, H., Wang, K., Yang, J., et al. (2019). The construction and analysis of ceRNA networks in invasive breast cancer: a study based on the cancer genome atlas. Cancer Manag. Res. 11, 1-11. doi: 10.2147/CMAR.S182521

Gerstberger, S., Hafner, M., and Tuschl, T. (2014). A census of human RNAbinding proteins. Nat. Rev. Genet. 15 (12), 829-845. doi: 10.1038/nrg3813

Goto-Yamaguchi, L., Yamamoto-Ibusuki, M., Yamamoto, Y., Fujiki, Y., Tomiguchi, M., Sueta, A., et al. (2018). Therapeutic predictors of neoadjuvant endocrine therapy response in estrogen receptor-positive breast cancer with reference to optimal gene expression profiling. Breast Cancer Res. Treat 172 (2), 353-362. doi: 10.1007/s10549-018-4933-5

Grammatikakis, I., Abdelmohsen, K., and Gorospe, M. (2017). Posttranslational control of HuR function. Wiley Interdiscip. Reviews-RNA 8 (1), 180239. doi: 10.1002/wrna. 1372

Gu, J., Chen, J., Feng, J., Liu, Y. F., Xue, Q., Mao, G. X., et al. (2016). Overexpression of ADAMTS5 can regulate the migration and invasion of non-small cell lung cancer. Tumor Biol. 37 (7), 8681-8689. doi: 10.1007/ s13277-015-4573-x
Han, L., Liu, B., Jiang, L., Liu, J., and Han, S. (2016). MicroRNA-497 downregulation contributes to cell proliferation, migration, and invasion of estrogen receptor alpha negative breast cancer by targeting estrogen-related receptor alpha. Tumour Biol. 37 (10), 13205-13214. doi: 10.1007/s13277-0165200-1

Hentze, M. W., Castello, A., Schwarzl, T., and Preiss, T. (2018). A brave new world of RNA-binding proteins. Nat. Rev. Mol. Cell Biol. 19 (5), 327-341. doi: $10.1038 /$ nrm.2017.130

Huang da, W., Sherman, B. T., and Lempicki, R. A. (2009). Bioinformatics enrichment tools: paths toward the comprehensive functional analysis of large gene lists. Nucleic Acids Res. 37 (1), 1-13. doi: 10.1093/nar/gkn923

Huang, D. W., Sherman, B. T., and Lempicki, R. A. (2009). Systematic and integrative analysis of large gene lists using DAVID bioinformatics resources. Nat. Protoc. 4 (1), 44-57. doi: 10.1038/nprot.2008.211

Huang, J., Sun, Y., Chen, H., Liao, Y., Li, S., Chen, C., et al. (2019). ADAMTS5 acts as a tumor suppressor by inhibiting migration, invasion and angiogenesis in human gastric cancer. Gastric Cancer 22 (2), 287-301. doi: 10.1007/s10120018-0866-2

Iorio, M. V., Ferracin, M., Liu, C. G., Veronese, A., Spizzo, R., Sabbioni, S., et al. (2005). MicroRNA gene expression deregulation in human breast cancer. Cancer Res. 65 (16), 7065-7070. doi: 10.1158/0008-5472.Can-05-1783

Jiang, C. J., Li, Y. S., Zhao, Z., Lu, J. P., Chen, H., Ding, N., et al. (2016a). Identifying and functionally characterizing tissue-specific and ubiquitously expressed human IncRNAs. Oncotarget 7 (6), 7120-7133. doi: 10.18632/ oncotarget.6859

Jiang, Q., He, M., Guan, S., Ma, M., Wu, H., Yu, Z., et al. (2016b). MicroRNA-100 suppresses the migration and invasion of breast cancer cells by targeting FZD-8 and inhibiting Wnt/beta-catenin signaling pathway. Tumour Biol. 37 (4), 5001-5011. doi: 10.1007/s13277-015-4342-x

Jiang, C., Ding, N., Li, J., Jin, X., Li, L., Pan, T., et al. (2018). Landscape of the long non-coding RNA transcriptome in human heart. Brief Bioinform. 20 (5), 1812 1825. doi: $10.1093 /$ bib/bby052

Kim, R. K., Suh, Y., Yoo, K. C., Cui, Y. H., Kim, H., Kim, M. J., et al. (2015). Activation of KRAS promotes the mesenchymal features of basal-type breast cancer. Exp. Mol. Med. 47, e137. doi: 10.1038/emm.2014.99

Konig, J., Zarnack, K., Luscombe, N. M., and Ule, J. (2012). Protein-RNA interactions: new genomic technologies and perspectives. Nat. Rev. Genet. 13 (2), $77-83$. doi: $10.1038 / \operatorname{nrg} 3141$

Latora, V., and Marchiori, M. (2001). Efficient behavior of small-world networks. Phys. Rev. Lett. 87 (19), 198701. doi: 10.1103/PhysRevLett.87.198701

Lee, J. D., Hempel, N., Lee, N. Y., and Blobe, G. C. (2010). The type III TGF-beta receptor suppresses breast cancer progression through GIPC-mediated inhibition of TGF-beta signaling. Carcinogenesis 31 (2), 175-183. doi: 10.1093/carcin/bgp271

Li, C. H., and Chen, Y. C. (2013). Targeting long non-coding RNAs in cancers: progress and prospects. Int. J. Biochem. Cell Biol. 45 (8), 1895-1910. doi: 10.1016/j.biocel.2013.05.030

Li, J. H., Liu, S., Zhou, H., Qu, L. H., and Yang, J. H. (2014a). starBase v2.0: decoding miRNA-ceRNA, miRNA-ncRNA and protein-RNA interaction networks from large-scale CLIP-Seq data. Nucleic Acids Res. 42 (Database issue), D92-D97. doi: 10.1093/nar/gkt1248

Li, Q., Yao, Y., Eades, G., Liu, Z., Zhang, Y., and Zhou, Q. (2014b). Downregulation of miR-140 promotes cancer stem cell formation in basallike early stage breast cancer. Oncogene 33 (20), 2589-2600. doi: 10.1038/ onc.2013.226

Li, J., Han, L., Roebuck, P., Diao, L., Liu, L., Yuan, Y., et al. (2015). TANRIC: an interactive open platform to explore the function of lncRNAs in cancer. Cancer Res. 75 (18), 3728-3737. doi: 10.1158/0008-5472.CAN-15-0273

Li, M., Li, D., Tang, Y., Wu, F., and Wang, J. (2017). CytoCluster: a cytoscape plugin for cluster analysis and visualization of biological networks. Int. J. Mol. Sci. 18 (9), 1880. doi: 10.3390/ijms18091880

Li, X., Yu, X., He, Y., Meng, Y., Liang, J., Huang, L., et al. (2018a). Integrated analysis of MicroRNA (miRNA) and mRNA profiles reveals reduced correlation between microRNA and target gene in cancer. BioMed. Res. Int. 2018, 1972606. doi: 10.1155/2018/1972606

Li, Y., Li, L., Wang, Z., Pan, T., Sahni, N., Jin, X., et al. (2018b). LncMAP: pancancer atlas of long noncoding RNA-mediated transcriptional network perturbations. Nucleic Acids Res. 46 (3), 1113-1123. doi: 10.1093/nar/gkx1311 
Li, Y. X., Yu, Z. W., Jiang, T., Shao, L. W., Liu, Y., Li, N., et al. (2018c). SNCA, a novel biomarker for Group 4 medulloblastomas, can inhibit tumor invasion and induce apoptosis. Cancer Sci. 109 (4), 1263-1275. doi: 10.1093/nar/ gkx1311

Liu, S. L., Patel, S. H., Ginestier, C., Ibarra, I., Martin-Trevino, R., Bai, S. M., et al. (2012). MicroRNA93 regulates proliferation and differentiation of normal and malignant breast stem cells. PloS Genet. 8 (6), e1002751. doi: 10.1371/ journal.pgen.1002751

Liu, H. R., Li, J., Koirala, P., Ding, X. F., Chen, B. H., Wang, Y. H., et al. (2016). Long non-coding RNAs as prognostic markers in human breast cancer. Oncotarget 7 (15), 20584-20596. doi: 10.18632/oncotarget.7828

Liu, A. N., Qu, H. J., Gong, W. J., Xiang, J. Y., Yang, M. M., and Zhang, W. (2019). LncRNA AWPPH and miRNA-21 regulates cancer cell proliferation and chemosensitivity in triple-negative breast cancer by interacting with each other. J. Cell Biochem. 120 (9), 14860-14866. doi: 10.1002/jcb.28747

Lo, P. H., Tanikawa, C., Katagiri, T., Nakamura, Y., and Matsuda, K. (2015). Identification of novel epigenetically inactivated gene PAMR1 in breast carcinoma. Oncol. Rep. 33 (1), 267-273. doi: 10.3892/or.20143581

Luo, M., Li, Z. W., Wang, W., Zeng, Y. G., Liu, Z. H., and Qiu, J. X. (2013). Long non-coding RNA H19 increases bladder cancer metastasis by associating with EZH2 and inhibiting E-cadherin expression. Cancer Lett. 333 (2), 213-221. doi: 10.1016/j.juro.2013.08.057

Mendoza-Rodriguez, M. G., Ayala-Sumuano, J. T., Garcia-Morales, L., ZamudioMeza, H., Perez-Yepez, E. A., and Meza, I. (2019). IL-1 beta inflammatory cytokine-induced TP63 isoform delta NP63 alpha signaling cascade contributes to cisplatin resistance in human breast cancer cells. Int. J. Mol. Sci. 20 (2), 270. doi: 10.3390/ijms20020270

Mercer, T. R., Dinger, M. E., Sunkin, S. M., Mehler, M. F., and Mattick, J. S. (2008). Specific expression of long noncoding RNAs in the mouse brain. Proc. Natl. Acad. Sci. U.S.A. 105 (2), 716-721. doi: 10.1073/pnas.0706729105

Mertins, P., Mani, D. R., Ruggles, K. V., Gillette, M. A., Clauser, K. R., Wang, P., et al. (2016). Proteogenomics connects somatic mutations to signalling in breast cancer. Nature 534 (7605), 55-62. doi: 10.1038/nature18003

Mi, H., and Thomas, P. (2009). PANTHER pathway: an ontology-based pathway database coupled with data analysis tools. Methods Mol. Biol. 563, 123-140. doi: 10.1007/978-1-60761-175-2_7

Milanovic, R., Roje, Z., Korusic, A., Deno, I. T., Baric, A., and Stanec, Z. (2013). Clinical and patohistological factors affecting the 5 year survival rate in a population of croatian women with invasive ductal breast carcinoma. Collegium Antropologicum 37 (2), 459-464.

Niknafs, Y. S., Han, S., Ma, T., Speers, C., Zhang, C., Wilder-Romans, K., et al. (2016). The lncRNA landscape of breast cancer reveals a role for DSCAM-AS1 in breast cancer progression. Nat. Commun. 7, 12791. doi: 10.1038/ ncomms 12791

Nishida, K., Kuwano, Y., Nishikawa, T., Masuda, K., and Rokutan, K. (2017). RNA binding proteins and genome integrity. Int. J. Mol. Sci. 18 (7), 1341. doi: 10.3390/ijms18071341

Pan, D. Z., Garske, K. M., Alvarez, M., Bhagat, Y. V., Boocock, J., Nikkola, E., et al. (2018). Integration of human adipocyte chromosomal interactions with adipose gene expression prioritizes obesity-related genes from GWAS. Nat. Commun. 9, 1512. doi: 10.1038/s41467-018-03554-9

Papageorgis, P., Ozturk, S., Lambert, A. W., Neophytou, C. M., Tzatsos, A., Wong, C. K., et al. (2015). Targeting IL13Ralpha2 activates STAT6-TP63 pathway to suppress breast cancer lung metastasis. Breast Cancer Res. 17, 98. doi: 10.1186/ s13058-015-0607-y

Plaisier, C. L., Pan, M., and Baliga, N. S. (2012). A miRNA-regulatory network explains how dysregulated miRNAs perturb oncogenic processes across diverse cancers. Genome Res. 22 (11), 2302-2314. doi: 10.1101/gr.133991.111

Ponting, C. P., Oliver, P. L., and Reik, W. (2009). Evolution and functions of long noncoding RNAs. Cell 136 (4), 629-641. doi: 10.1016/j.cell.2009.02.006

Rana, T. M. (2007). Illuminating the silence: understanding the structure and function of small RNAs. Nat. Rev. Mol. Cell Biol. 8 (1), 23-36. doi: 10.1038/ nrm 2085

Roscigno, G., Quintavalle, C., Donnarumma, E., Puoti, I., Diaz-Lagares, A., Iaboni, M., et al. (2016). MiR-221 promotes stemness of breast cancer cells by targeting DNMT3b. Oncotarget 7 (1), 580-592. doi: 10.18632/oncotarget.5979

Sahay, D., Leblanc, R., Grunewald, T. G. P., Ambatipudi, S., Ribeiro, J., Clezardin, P., et al. (2015). The LPA1/ZEB1/miR-21-activation pathway regulates metastasis in basal breast cancer. Oncotarget 6 (24), 20604-20620. doi: 10.18632 /oncotarget. 3774

Salem, O., Erdem, N., Jung, J., Munstermann, E., Worner, A., Wilhelm, H., et al. (2016). The highly expressed 5'isomiR of hsa-miR-140-3p contributes to the tumor-suppressive effects of miR-140 by reducing breast cancer proliferation and migration. BMC Genomics 17, 566. doi: 10.1186/s12864-016-2869-x

Schmitt, A. M., and Chang, H. Y. (2016). Long noncoding RNAs in cancer pathways. Cancer Cell 29 (4), 452-463. doi: 10.1016/j.ccell.2016.03.010

Serra-Musach, J., Aguilar, H., Iorio, F., Comellas, F., Berenguer, A., Brunet, J., et al. (2012). Cancer develops, progresses and responds to therapies through restricted perturbation of the protein-protein interaction network. Integr. Biol. (Camb) 4 (9), 1038-1048. doi: 10.1039/c2ib20052j

Shannon, P., Markiel, A., Ozier, O., Baliga, N. S., Wang, J. T., Ramage, D., et al. (2003). Cytoscape: a software environment for integrated models of biomolecular interaction networks. Genome Res. 13 (11), 2498-2504. doi: 10.1101/gr.1239303

Siegel, R. L., Miller, K. D., and Jemal, A. (2017). Cancer Statistics, 2017. Ca- $a$ Cancer J. Clin. 67 (1), 7-30. doi: 10.3322/caac.21387

Singh, R., Yadav, V., Kumar, S., and Saini, N. (2015). MicroRNA-195 inhibits proliferation, invasion and metastasis in breast cancer cells by targeting FASN, HMGCR, ACACA and CYP27B1. Sci. Rep. 5, 17454. doi: 10.1038/ srep 17454

Slattery, M. L., John, E. M., Stern, M. C., Herrick, J., Lundgreen, A., Giuliano, A. R., et al. (2013). Associations with growth factor genes (FGF1, FGF2, PDGFB, FGFR2, NRG2, EGF, ERBB2) with breast cancer risk and survival: the breast cancer health disparities study. Breast Cancer Res. Treat 140 (3), 587-601. doi: 10.1007/s10549-013-2644-5

Song, L. L., Chang, R. X., Dai, C., Wu, Y. J., Guo, J. Y., Qi, M. Y., et al. (2017). SORBS1 suppresses tumor metastasis and improves the sensitivity of cancer to chemotherapy drug. Oncotarget 8 (6), 9108-9122. doi: 10.18632/ oncotarget.12851

Stewart, B. W., and Wild, C. (2014). International agency for research on cancer, and world health organization World cancer report 2014.

Subramanian, A., Tamayo, P., Mootha, V. K., Mukherjee, S., Ebert, B. L., Gillette, M. A., et al. (2005). Gene set enrichment analysis: a knowledge-based approach for interpreting genome-wide expression profiles. Proc. Natl. Acad. Sci. U. S. A. 102 (43), 15545-15550. doi: 10.1073/pnas.0506580102

Sun, M., Wu, D., Zhou, K., Li, H., Gong, X., Wei, Q., et al. (2019). An eightlncRNA signature predicts survival of breast cancer patients: a comprehensive study based on weighted gene co-expression network analysis and competing endogenous RNA network. Breast Cancer Res. Treat 175 (1), 59-75. doi: 10.1007/s10549-019-05147-6

Tang, X., Tu, G., Yang, G. L., Wang, X., Kang, L. M., Yang, L. P., et al. (2019). Autocrine TGF-beta 1/miR-200s/miR-221/DNMT3B regulatory loop maintains CAF status to fuel breast cancer cell proliferation. Cancer Lett. 452, 79-89. doi: 10.1016/j.canlet.2019.02.044

Tang, G. L. (2005). siRNA and miRNA: an insight into RISCs. Trends Biochem. Sci. 30 (2), 106-114. doi: 10.1016/j.tibs.2004.12.007

Uhlen, M., Fagerberg, L., Hallstrom, B. M., Lindskog, C., Oksvold, P., Mardinoglu, A., et al. (2015). Proteomics. tissue-based map of the human proteome. Science 347 (6220), 1260419. doi: 10.1126/science.1260419

Wagner, A., and Fell, D. A. (2001). The small world inside large metabolic networks. Proc. R. Soc. B-Biolog. Sci. 268 (1478), 1803-1810. doi: 10.1098/ rspb.20011711

Wang, J., Liu, Q., and Shyr, Y. (2015). Dysregulated transcription across diverse cancer types reveals the importance of RNA-binding protein in carcinogenesis. BMC Genomics 16, S5. doi: 10.1186/1471-2164-16-S7-S5

Wang, L., Jiang, C. F., Li, D. M., Ge, X., Shi, Z. M., Li, C. Y., et al. (2016a). MicroRNA-497 inhibits tumor growth and increases chemosensitivity to 5fluorouracil treatment by targeting KSR1. Oncotarget 7 (3), 2660-2671. doi: 10.18632/oncotarget6545

Wang, N., Chen, P., Huang, L. P., and Wang, T. Z. (2016b). Prognostic significance of microRNA-10b overexpression in breast cancer: a meta-analysis. Genet. Mol. Res. 15 (2), gmr7350. doi: 10.4238/gmr.15027350

Wang, Y., Zhang, X., Zou, C., Kung, H. F., Lin, M. C., Dress, A., et al. (2016c). miR195 inhibits tumor growth and angiogenesis through modulating IRS1 in breast cancer. BioMed. Pharmacother. 80, 95-101. doi: 10.1016/ j.biopha.2016.03.007 
Wang, C. Q., Chen, L., Dong, C. L., Song, Y., Shen, Z. P., Shen, W. M., et al. (2017). MiR-377 suppresses cell proliferation and metastasis in gastric cancer via repressing the expression of VEGFA. Eur. Rev. Med. Pharmacol. Sci. 21 (22), 5101-5111. doi: 10.26355/eurrev_201711_13826

Wang, D. Y., Gendoo, D. M. A., Ben-David, Y., Woodgett, J. R., and Zacksenhaus, E. (2019a). A subgroup of microRNAs defines PTEN-deficient, triple-negative breast cancer patients with poorest prognosis and alterations in RB1, MYC, and Wnt signaling. Breast Cancer Res. 21 (1), 18. doi: 10.1186/s13058-0191098-z

Wang, X., Chen, T., Zhang, Y., Zhang, N., Li, C., Li, Y., et al. (2019b). Long noncoding RNA Linc00339 promotes triple-negative breast cancer progression through miR-377-3p/HOXC6 signaling pathway. J. Cell Physiol. 234 (8), 13303-13317. doi: 10.1002/jcp.28007

Wei, Y. T., Guo, D. W., Hou, X. Z., and Jiang, D. Q. (2017). miRNA-223 suppresses FOXO1 and functions as a potential tumor marker in breast cancer. Cell Mol. Biol. (Noisy-le-grand) 63 (5), 113-118. doi: 10.14715/cmb/ 2017.63.5.21

Weigelt, B., Horlings, H. M., Kreike, B., Hayes, M. M., Hauptmann, M., Wessels, L. F. A., et al. (2008). Refinement of breast cancer classification by molecular characterization of histological special types. J. Pathol. 216 (2), 141-150. doi: $10.1002 /$ path2407

Wen, J. X., Li, X. Q., and Chang, Y. (2018). Signature gene identification of cancer occurrence and pattern recognition. J. Comput. Biol. 25 (8), 907-916. doi: $10.1089 / \mathrm{cmb} .20170261$

Wu, W., Wagner, E. K., Hao, Y., Rao, X., Dai, H., Han, J., et al. (2016a). Tissuespecific co-expression of long non-coding and coding RNAs associated with breast cancer. Sci. Rep. 6, 32731. doi: 10.1038/srep32731

Wu, Z., Cai, X., Huang, C., Xu, J., and Liu, A. (2016b). miR-497 suppresses angiogenesis in breast carcinoma by targeting HIF-lalpha. Oncol. Rep. 35 (3), 1696-1702. doi: 10.3892/or.20154529

Xiao, B., Zhang, W., Chen, L., Hang, J., Wang, L., Zhang, R., et al. (2018). Analysis of the miRNA-mRNA-lncRNA network in human estrogen receptor-positive and estrogen receptor-negative breast cancer based on TCGA data. Gene 658, 28-35. doi: 10.1016/j.gene.2018.03.011

Yan, L. X., Huang, X. F., Shao, Q., Huang, M. Y., Deng, L., Wu, Q. L., et al. (2008). MicroRNA miR-21 overexpression in human breast cancer is associated with advanced clinical stage, lymph node metastasis and patient poor prognosis. RNA 14 (11), 2348-2360. doi: 10.1261/rna.1034808

Yan, X., Hu, Z., Feng, Y., Hu, X., Yuan, J., Zhao, S. D., et al. (2015). Comprehensive genomic characterization of long non-coding RNAs across human cancers. Cancer Cell 28 (4), 529-540. doi: 10.1016/j.ccell.2015.09.006

Yan, Y. L., Xu, Z. J., Hu, X. F., Qian, L., Li, Z., Zhou, Y. Y., et al. (2018). SNCA is a functionally low-expressed gene in lung adenocarcinoma. Genes 9 (1), 16. 10.3390/genes9010016

Yang, G. D., Lu, X. Z., and Yuan, L. J. (2014). LncRNA: a link between RNA and cancer. Biochim. Et Biophys. Acta-Gene Regul. Mech. 1839 (11), 1097-1109. doi: 10.1016/j.bbagrm.2014.08.012

Yang, R., Xing, L., Wang, M., Chi, H., Zhang, L., and Chen, J. (2018a). Comprehensive analysis of differentially expressed profiles of lncRNAs/ mRNAs and miRNAs with associated ceRNA networks in triple-negative breast cancer. Cell Physiol. Biochem. 50 (2), 473-488. doi: 10.1159/000494162
Yang, Y., Yang, H., Xu, M., Zhang, H., Sun, M., Mu, P., et al. (2018b). Long noncoding RNA (lncRNA) MAGI2-AS3 inhibits breast cancer cell growth by targeting the Fas/FasL signalling pathway. Hum. Cell 31 (3), 232-241. doi: 10.1007/s13577-018-0206-1

Yanwirasti, W.,. A. H., and Arisanty, D. (2017). Evaluation of MiR-21 and MiR$10 \mathrm{~b}$ expression of human breast cancer in west sumatera. Pak J. Biol. Sci. 20 (4), 189-196. doi: 10.3923/pjbs.2017.189.196

Yi, C. H., Zheng, T., Leaderer, D., Hoffman, A., and Zhu, Y. (2009). Cancer-related transcriptional targets of the circadian gene NPAS2 identified by genome-wide ChIP-on-chip analysis. Cancer Lett. 284 (2), 149-156. doi: 10.1016/ j.canlet.2009.04.017

Yin, Y., Shen, C., Xie, P., Cheng, Z., and Zhu, Q. (2016). Construction of an initial microRNA regulation network in breast invasive carcinoma by bioinformatics analysis. Breast 26, 1-10. doi: 10.1016/j.breast.2015.11.008

Yu, L., Lu, Y., Han, X., Zhao, W., Li, J., Mao, J., et al. (2016). microRNA -140-5p inhibits colorectal cancer invasion and metastasis by targeting ADAMTS5 and IGFBP5. Stem Cell Res. Ther. 7 (1), 180. doi: 10.1186/s13287-016-0438-5

Zang, H., Li, N., Pan, Y., and Hao, J. (2017). Identification of upstream transcription factors (TFs) for expression signature genes in breast cancer. Gynecol. Endocrinol. 33 (3), 193-198. doi: 10.1080/09513590.2016.1239253

Zhang, J., Fan, D., Jian, Z., Chen, G. G., and Lai, P. B. (2015). Cancer specific long noncoding RNAs show differential expression patterns and competing endogenous RNA potential in hepatocellular carcinoma. PloS One 10 (10), e0141042. doi: 10.1371/journal.pone.0141042

Zhou, M., Wang, X. J., Shi, H. B., Cheng, L., Wang, Z. Z., Zhao, H. Q., et al. (2016a). Characterization of long non-coding RNA-associated ceRNA network to reveal potential prognostic lncRNA biomarkers in human ovarian cancer. Oncotarget 7 (11), 12598-12611. doi: 10.18632/oncotarget7181

Zhou, M., Zhong, L., Xu, W., Sun, Y., Zhang, Z., Zhao, H., et al. (2016b). Discovery of potential prognostic long non-coding RNA biomarkers for predicting the risk of tumor recurrence of breast cancer patients. Sci. Rep. 6, 31038. doi: 10.1038/srep31038

Zhu, L., Xi, P. W., Li, X. X., Sun, X., Zhou, W. B., Xia, T. S., et al. (2019a). The RNA binding protein RBMS3 inhibits the metastasis of breast cancer by regulating Twistl expression. J. Exp. Clin. Cancer Res. 38 (1), 105. doi: 10.1186/s13046019-1111-5

Zhu, M., Wang, X., Gu, Y., Wang, F., Li, L., and Qiu, X. (2019b). MEG3 overexpression inhibits the tumorigenesis of breast cancer by downregulating miR-21 through the PI3K/Akt pathway. Arch. Biochem. Biophys. 661, 22-30. doi: 10.1016/j.abb.2018.10.021

Conflict of Interest: The authors declare that the research was conducted in the absence of any commercial or financial relationships that could be construed as a potential conflict of interest.

Copyright (c) 2020 Dong, Xiao, Shi and Jiang. This is an open-access article distributed under the terms of the Creative Commons Attribution License (CC BY). The use, distribution or reproduction in other forums is permitted, provided the original author (s) and the copyright owner(s) are credited and that the original publication in this journal is cited, in accordance with accepted academic practice. No use, distribution or reproduction is permitted which does not comply with these terms. 